\title{
Modeling of Sokoto Daily Average Temperature: A Fractional Integration Approach
}

\author{
${ }^{* 1}$ L.K. Ibrahim, ${ }^{2}$ B.K. Asare, ${ }^{2}$ M. Yakubu and ${ }^{1}$ U. Dauda \\ ${ }^{1}$ Department of Mathematics and Computer Science, Umaru Musa Yar'adua University, Katsina, Nigeria. \\ ${ }^{2}$ Department of mathematics Usmanu Danfodiyo University, Sokoto \\ [Corresponding Author: ykmtm2000@yahoo.com]
}

\begin{abstract}
Autoregressive fractional integrated moving average modeling strategy was used to model the daily average temperature (DAT) series of Sokoto metropolis for the period of 01/01/2003 to 03/04/2007. The time plot suggests that there is persistence dependence in the series. The order of fractional integration was found to be 0.6238841 . The correct model for the daily average temperature data (DAT) of Sokoto metropolis was built. Two models were found to be more adequate for describing, explaining and forecasting the temperature, $\operatorname{ARFIMA}(3,0.6238841,1)$ and $\operatorname{ARFIMA}(1,0.6238841,3)$. But by checking the forecastability, ARFIMA $(3,0.6238841,1)$ model was found to be the best optimal model that will best forecast Sokoto metropolis temperature. The fitted model should be used for future forecast of temperature of Sokoto metropolis. Forecasting temperature is important to Agriculturist, Geographers and Hydrologist. Air temperature determines the rate of evapotranspiration.

Keywords: Fractional Integration, ARFIMA, Temperature and Sokoto.
\end{abstract}

\section{INTRODUCTION}

Sokoto is a city located in the extreme northwest of Nigeria. The location of Sokoto in Nigeria is at Latitude $13^{0} 02 \mathrm{~N}$ and Longitude $05^{\circ} 15 \mathrm{E}$. Sokoto State is in the dry Sahel, surrounded by sandy Savannah and isolated hills. Sokoto as a whole is very hot area. The warmest months are February to April when daytime temperature is rising. The raining season is from June to October during which showers are a daily occurrence. From late October to February, during the cold season, the climate is dominated by the Hamattan wind blowing Sahara dust over the land. The dust dims the sunlight there by lowering temperatures significantly and also leading to the inconvenience of dust everywhere in houses.

Research on long memory and fractionally integrated processes has continued at an accelerating rate since the initial publication of the work of Granger (1980); Granger and Joyeux (1980) and Hosking (1981) which parameterized the processes of Hurst (1951) on the time series with hyperbolically decaying autocorrelations (Baillie, 2007). The long memory or long term dependence property describes the high-order correlation structure of a series. If a series exhibits long memory, there is persistent temporal dependence even between distant observations. ñSuch series are characterized by distinct but non- periodic cyclical patternsò. Fractionally integrated processes can give rise to long memory (Beran, 1994).

The ARFIMA (p,d,q) model was introduced by Granger and Joyeux (1980). Since then there has been great strides in the estimation of long memory modelling, Granger and Joyeux (1980); Hoskin (1981); Geweke and Portter-Hudack (1983); Sowel (1992) and Mayoral (2007).

Fractional Integration is part of the larger classification of time series, commonly referred to as đóng memoryô models. However, recent empirical evidence suggests that temperature series may be well described in terms of fractionally integrated processes (Gil-Alana, 2009).

Fractionally integrated $\mathrm{I}(\mathrm{d})$ processes have attracted growing attention among empirical researchers. In fact this is because $\mathrm{I}(\mathrm{d})$ processes provide an extension to the classical dichotomy of $\mathrm{I}(0)$ and $\mathrm{I}(1)$ time series and equip us with more general alternatives in long range dependence (Shimotsu, 2002). Empirical research continues to find evidence that $\mathrm{I}(\mathrm{d})$ processes can provide a suitable description of certain long range characteristics (Henry and Zaffaroni, 2002). The class of fractionally integrated processes is an 
extension of the class of ARIMA processes stemming from Box and Jenkins methodology. One of their originalities is the explicit modeling of the long term correlation structure (Diebolt and Guiraud, 2000). Autoregressive fractionally integrated moving average models grant social scientist greater freedom and flexibility in modeling long memory processes, for example, modeling data as fractionally integrated allows the researchers to model slower rates of decay than with other common techniques such as ARMA or ARIMA models (Baillie, 1996).

The daily maximum and minimum temperatures in Melbourne, Australia, for the period 1981ї 1990 were examined by means of fractional integration techniques. Using a parametric testing procedure (Robinson 1994), the results show that the time series for both datasets can be specified in terms of fractionally integrated statistical models: the maximum temperatures with orders of integration slightly smaller than 0.5 and the minimum temperatures with values slightly higher. Thus, the temperatures seem to be stationary in the case of the maximum values and non-stationary for the minimum ones, but mean reverting in both cases. Moreover, no significant trends in the model were found, implying that there is no evidence of climatic change in the data (Gil-Alana, 2004).

However, in Africa and more especially in Nigeria little or not much has been done in this area.

The objectives of this study is, therefore, to use a battery of statistical techniques to study the daily average temperature of Sokoto metropolis and use autoregressive fractionally integrated moving average (AFRIMA) processes to build a model for the Sokoto daily average temperature.

\section{MATERIALS AND METHODS}

Arfima (p, d, q) Model: The general Fractionally Integrated Autoregressive Moving Average [ARFIMA (p, d, q)] model is:

$$
\Phi(B)(1-B)^{d} X(t)=\Theta(B) \varepsilon(t)
$$

Where $B$ is the lag operators $\Phi(B)$ and $\Theta(B)$ are polynomials of orders $\mathrm{p}$ and $\mathrm{q}$ respectively with $\Phi_{0}=-1$ and $\Theta_{0}=1$.

Box and Jenkins (1976) cited that the model should be parsimonious. Therefore, they recommended the need to use as few model parameters as possible so that the model fulfils all the diagnostic checks. Akaike (1974) suggests a mathematical formulation of the parsimony criterion of model building as AIC (Akaike Information Criterion) for the purpose of selecting an optimal model fits to a given data. Mathematical formulation of AIC is defined as:

$$
\operatorname{AIC}(\mathrm{M})=\ln \widehat{\sigma}_{\varepsilon_{t}}^{2}+2 M
$$

Where $\mathrm{M}$ is the number of $\mathrm{AR}$ and $\mathrm{MA}$ parameters to estimate. The model that gives the minimum AIC is selected as a parsimonious model (Yurekli, 2007).

\section{Fractionally Integrated Processes}

The class of fractional integrated processes is an extension of the class of ARIMA processes, stemming from Box and Jenkins methodology. One of their originalities is the explicit modeling of the long term correlation structure. According to the values of parameters, these processes will possess the long range dependence property or long memory introduced by Hurst (1951) and Mandelbrot and Van Ness. (1968).

Let $X_{t}, t=1,2, \ldots \ldots . n$ be a time series and be $\rho(k)$ its autocorrelation function:

$$
\rho(k)=E\left(X_{t}, X_{t-k}\right)
$$

The stationary property is verified if:

$$
\sum_{k=0}^{\infty} \rho(k)^{2}<\infty
$$

In this case, it is said that $X_{t}$ has the long memory property if:

$$
\sum_{k=0}^{\infty} / \rho(k) /<\infty
$$

A way of representing such correlation structures is the use of fractional integrated processes. These models are defined from the fractional differentiation operator $[1-\beta]^{d}$. The fractional operator is broken down using a binomial series:

$$
[1-\beta]^{d}=1-d \beta-\ldots-\frac{\Gamma(k-d)}{\Gamma(k+1) \Gamma(-d)} \beta^{k}+\mathrm{O}\left(\beta^{k+1}\right)
$$

This operator makes it possible to define fractional integrated processes. It is assumed that process $x_{t} t=1 \ldots n$ (assumed to be centered for the purpose of simplicity, $E[x]=0$ ), follows an 
Auto Regressive Fractional Integrated Moving Average (ARFIMA) process if:

$$
[1-\beta]^{d} X_{t}=\mu_{t}
$$

Where $\mu_{t}$ is a usual $\operatorname{ARMA}(\mathrm{p}, \mathrm{q})$ process,

$$
\phi_{p}(\beta) \mu_{t}=\theta_{q}(\beta) \mathcal{E}_{t},
$$

$\varepsilon_{t}$ is a white noise with zero mean and variance $\sigma_{\varepsilon}^{2}$.We assume that $\mu_{t}$ verifies the stationarity and invertibility conditions. This assumption is necessary to establish the following properties. One can demonstrate that:

AThe process $X_{t}$ is stationary if $d<1 / 2$,

ÂThe process $X_{t}$ is invertible if $d>-1 / 2$. Odaki (1993) spread this interval to $d>-1$ by using a weak invertibility concept.

$\AA$ The stationary process $X_{t}$ will have long memory if $0<\mathrm{d}<1 / 2$.

The degree of persistence of the series can be measured through a fractional differencing parameter (Gil-Alana, 2009).

Test and estimation of order of Integration: There exist several procedures for estimating the fractional differencing parameter in semi parametric contexts. Of these, the logperiodogram regression estimate proposed by Geweke and Porter-Hudak (1983) has been the most widely used (Shimotsu et al., 2002).

Given a fractional integrated process $\left\{\mathrm{Y}_{\mathrm{t}}\right\}$, its spectral density is given by

$$
f(\omega)=[2 \sin (\omega / 2)]^{-2 d} f(\omega)
$$

Where $\omega$ is the Fourier frequency, $f_{u}(\omega)$ is the spectral density corresponding to $u_{t}$ and $u_{t}$ is a stationary short memory disturbance with zero mean.

The fractional differencing parameter $\mathrm{d}$ can be estimated by the regression equations constructed from $p(\omega \leq X)=1+2 \sum_{j=1}^{\infty}\left(1-4 X^{2} j^{2}\right) e^{-2 X^{2} j^{2}}$

GPH showed that using a periodogram estimate of $f\left(\omega_{j}\right)$, if the number of frequencies $m$ used is a function $\mathrm{g}(\mathrm{n})$ (a positive integer) of the sample size $\mathrm{n}$ where $\mathrm{m}=g(n)=n^{\alpha}$ with $0<\alpha<1$, it can be demonstrated that the least squares estimate $\hat{d}$ using the above regression is asymptotically normally distributed in large samples.

$$
\widehat{d} \sim N\left(d, \frac{\pi^{2}}{6 \sum_{j=1}^{g(n)}\left(U_{j}-\bar{U}\right)^{2}}\right)
$$

Where $U_{j}=\ln \left[4 \sin ^{2}\left(\omega_{j} / 2\right)\right]$ and $\bar{U}$ is the sample of $U_{j}, \mathrm{j}=1$ é é $\mathrm{g}(\mathrm{n})$.

Stationarity/Unit Root Test: The pattern and general behaviour of the series is examined from the time plot. The series was examined for stationarity, linearity and gaussianity. The tests for unit roots/stationarity are: Augmented Dickey ï Fuller test or the Phillips-Perron test and KPSS test proposed by Kwiatkowski et al., (1992) to test for the stationarity of a series.

The ADF test has the following model representation:

$\Delta y_{t}=\alpha y_{t-1}+x_{t} \delta+\beta_{1} \Delta y_{t-1}+\beta_{2} \Delta y_{t-2}+\ldots .+\Delta \beta_{p} y_{t-p}$

Where,

$\Delta y_{t}$ is the differenced series.

$y_{t-1}$ is the immediate previous observation.

$x_{t}$ is the optional exogenous regressor which may be constant, or a constant and trend. $\alpha$ and $\delta$ are parameters to be estimated. $\beta_{1}, \ldots, \beta_{p}$ is the coefficients of the lagged difference term up to lag $\mathrm{p}$.

Test statistic: $t_{\alpha}=\hat{\alpha} / \operatorname{se}(\hat{\alpha})$

The hypothesis testing:

$H_{0}: \alpha=0$ (The series contains unit roots)

$H_{1}: \alpha<0 \quad$ (The series is stationary)

Model Checking: After estimation of the model, the Boxï Jenkins model building strategy entails a diagnosis of the adequacy of the model. More specifically, it is necessary to ascertain in what way the model is adequate and in what way it is inadequate. This stage of the modeling strategy involves several steps Kendall and Ord (1990).

A good way to check model adequacy of an overall Box-Jenkins model is to analyze the 
residual obtained from the model. Two statistic(s) have suggested determining whether the first $K$ sample autocorrelation indicate adequacy of the model, that are, the Box-Pierce statistic and Ljung-Box statistic (portmanteau test). In spite of this, we can also check the model adequacy by examining the sample autocorrelation function of the residual (ACF) and sample partial autocorrelation function of the residual (PACF). We can conclude that the model is adequate if there are no spikes in the ACF and PACF (Azami, 2009). We can also use the Jarque-Bera test to test for normality of residual.

Softwares for the Analysis: The R, MINITAB and Gretl softwares ware used for the analysis.

Data and Data Analysis: The daily average temperature (DAT) series of Sokoto metropolis for the period 01/01/2003 to 03/04/2007 was used (Table 1). The data sets are obtained from Energy Research Center, Usmanu Danfodiyo University, Sokoto.

Table 1: Results of Summary Statistics for DAT in ${ }^{0} \mathrm{C}$

\begin{tabular}{ll}
\hline Parameter & Value \\
\hline Mean & 28.8306 \\
Median & 28.5000 \\
Minimum & 12.2500 \\
Maximum & 40.5000 \\
Std. Dev. & 4.22908 \\
CV & 0.146687 \\
Skewness & -0.0366816 \\
Ex. Kurtosis & -0.000971508 \\
\hline
\end{tabular}

The DAT and its graphical properties

Visual inspection of the plot of daily average temperature series in Figure1 reveals that short term random fluctuations, but long fluctuations of unequal duration are readily apparent. Long term or persistent dependence observed. The series is assumed to be non stationary.

Autocorrelation Function (ACF): A time series exhibits long memory when there is significant dependence between observations that are separated by a long period of time. Characteristic of a long memory time series is an autocorrelation function that decays hyperbolically to zero.
Figure 2 shows the plot of the sample autocorrelation function (ACF) of the daily average temperature series (DAT). Non periodic cycles, persistence and slow decay of ACF are observed, with relatively high concentration of mass at high lags which naturally is suggesting long memory model.

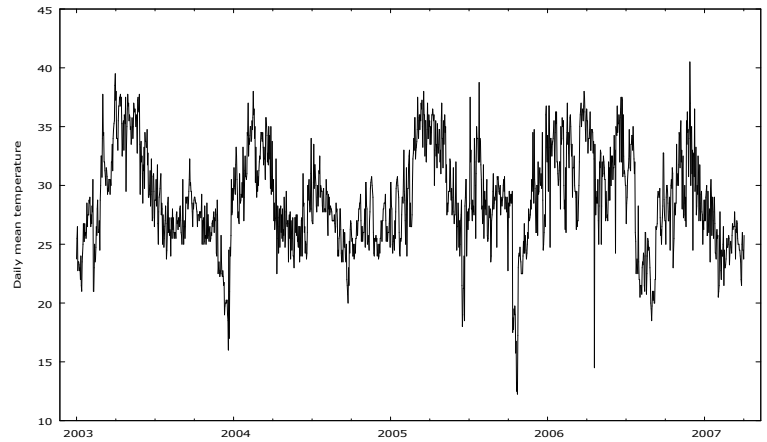

Figure: 1 Daily average temperature of Sokoto metropolis from 01/01/2003-03/04/2007 measured in ${ }^{0} \mathrm{C}$
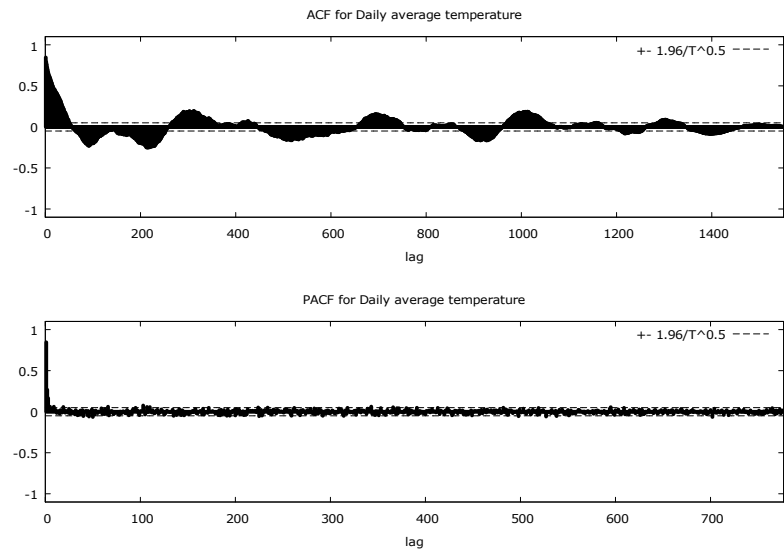

Figure 2: $\mathrm{ACF}$ and $\mathrm{PACF}$ for daily average temperature of Sokoto metropolis

Spectral Density Function: The frequency domain definition of long memory states that the spectral density function is unbounded at some frequency $\boldsymbol{x}$ in the interval [0,']. Persistence would be reflected in the spectral density function with a relatively high concentration of mass around zero frequency (Labato, 1997). Figure 3 gives the spectral density plot of the daily average temperature series of Sokoto metropolis with relatively high concentration of mass around zero frequency. 


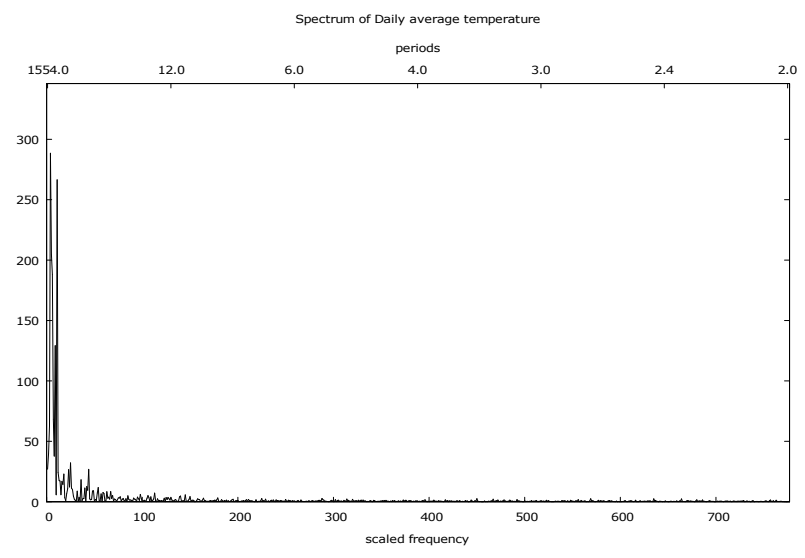

Figure.3:Spectrum of the daily average temperature $\left({ }^{0} \mathrm{C}\right)$ of Sokoto metropolis
Stationarity and Unit Root Test: The KPSS test statistic tests the null hypothesis of stationarity against the alternative of unit root and the decision rule is to accept the null hypothesis when the value of the test statistic is less than the critical value. The ADF statistic test the null hypothesis of presence of unit root against the alternative of no unit root and the decision rule is to reject the null hypothesis when the value of test statistic is less than the critical value. The result is shown in Table 2. According to the ADF test and KPSS test for the daily average temperature series, the results have shown that the time series is neither I (1) nor I (0). Further tests should be done to assume the order of differentiation.

Table 2: Results for ADF and KPSS Tests for DAT

\begin{tabular}{llcccccc}
\hline VARIABLE & \multicolumn{3}{c}{ CRITICAL LEVEL } & \multicolumn{3}{c}{ CRITICAL LEVEL } \\
& \multicolumn{3}{c}{ ADF } & \multicolumn{3}{c}{ KPSS } \\
\hline \multirow{4}{*}{ DAT } & $2.5 \%$ & $5 \%$ & $10 \%$ & $2.5 \%$ & $5 \%$ & $10 \%$ \\
& & -3.15 & -2.89 & -2.55 & 0.176 & 0.146 & 0.119 \\
& Lags & 0 & 2 & 4 & 0 & 2 & 4 \\
& TEST & -9.4683 & -5.6206 & -4.76877 & 1.71616 & 0.646226 & 0.40852 \\
\hline
\end{tabular}

Test and estimation of order of Integration: There exist many approaches for estimating and testing the fractional differencing parameter. Some of them are parametric and some semi parametric (Gil-Alana, 2004). In this research we implement one of the semi parametric techniques proposed by Geweke et al., (1983). The application of this test on the series allows us to test the null hypothesis of a unit root $(d=1)$ against the alternative of fractional integration $(d$ $<1)$. The fractional differencing parameter $\mathrm{d}$ is estimated by using the regression of equation (5). The value can be adjusted using the bandwidth parameter. Traditionally the number of bandwidth is chosen from the interval $\left[\mathrm{T}^{1 / 2}, \mathrm{~T}^{4 / 5}\right]$ (Robinson, 1994). However, Hurvich and Deo (1998) showed that the optimal bandwidth is $\left(\mathrm{T}^{0.8}\right)$. The bandwidth of 0.8 is chosen in this work and the result of the estimated fractional parameter $\mathrm{d}$ is given in Table 3.
Table 3: Results of GPH estimate for d parameter

\begin{tabular}{llll}
\hline Variable & d & Sd.as & Sd.reg \\
\hline DAT & 0.6238841 & 0.03579196 & 0.03758034 \\
\hline
\end{tabular}

Here $\boldsymbol{d}$ is the fractional differencing parameter, sd.as is the standard deviation and sd.reg is the standard error. The value of the differencing parameter $d$ is non integer and it is showing non stationary and mean reverting.

The value of the estimated $\boldsymbol{d}$ parameter is used in equation (1) to fractionally difference the data. Similar tests were performed on the fractionally differenced series, and observed that the new series is stationary. The results of the tests are given in Table 4 . The newly obtained set of data can be used to perform the long memory analysis, since all the fractionally integrated part has been removed. 
Ibrahim et al.: Modeling of Sokoto Daily Average Temperature: A Fractional Integration Approach

Table 4: Results of ADF and KPSS tests for the fractionally difference series

\begin{tabular}{lllllll}
\hline & \multicolumn{3}{c}{ CRITICAL LEVEL } & \multicolumn{3}{c}{ CRITICAL LEVEL } \\
KDPSS \\
\hline VARIABLES & $10 \%$ & $5 \%$ & $2.5 \%$ & $10 \%$ & $5 \%$ & $2.5 \%$ \\
& -2.57 & -2.89 & -3.15 & 0.347 & 0.463 & 0.574 \\
TEST STAT. & -12.5803 & & & 0.1089 & & \\
\hline
\end{tabular}

\section{ARFIMA Model Identification}

The value of the estimated fractional differencing parameter $\mathrm{d}$ is used to fractionally difference the data. The newly obtained set of data is now $\phi_{p}(\beta) \mu_{t}=\theta_{q}(\beta) \mathcal{E}_{t}$, which is $\operatorname{ARMA}(\mathrm{p}, \mathrm{q})$ series, and is taken and modeled as an ARMA (p, q) process since all the integrated part have been removed. The results of the models are in Table 5.

We selected three models with low AIC which is a common procedure in ARFIMA modeling
(Laurini et al, 2003) and find the best among them. The models selected are ARFIMA (3, $0.6238841,1)$, ARFIMA $(1,0.6238841,3)$ and ARFIMA $(1,0.6238841,2)$

Estimation of the Models: We estimate the parameters of ARFIMA (p, d, q) as the optimal model. Parameters are estimated by exact maximum likelihood method and the order of ARFIMA parameters are selected from Akaike information criteria and are given in Table 6.

Table 5: Results of Model identification for DAT

\begin{tabular}{lccc}
\hline Model & AIC & HQC & BIC \\
\hline ARFIMA(0,0.6238841, 0) & 7225.518 & 7229.496 & 7236.214 \\
ARFIMA(1,0.6238841, 0) & 7227.498 & 7233.465 & 7243.542 \\
ARFIMA(1,0.6238841, 1) & 7229.414 & 7237.370 & 7250.806 \\
ARFIMA(1,0.6238841, 2) & 7224.266 & 7234.211 & 7251.006 \\
ARFIMA(2,0.6238841, 1) & 7224.606 & 7234.551 & 7251.346 \\
ARFIMA(1,0.6238841, 3) & 7223.517 & 7235.450 & 7255.604 \\
ARFIMA(3,0.6238841, 1) & 7223.967 & 7235.900 & 7256.055 \\
ARFIMA(3,0.6238841, 2) & 7224.533 & 7238.455 & 7261.968
\end{tabular}

Table 6: Results of Estimated parameters of ARFIMA (p, d, q) models

\begin{tabular}{llll}
\hline PARAMETERS & ARFIMA (1, d, 2) & ARFIMA (1, d, 3) & ARFIMA (3, d, 1) \\
\hline$\widehat{\phi}_{1}$ & 0.700770 & 0.405175 & 0.363368 \\
$\widehat{\phi}_{2}$ & - & - & 0.0335849 \\
$\widehat{\phi}_{3}$ & - & -0.413688 & 0.0546663 \\
$\hat{\theta}_{1}$ & -0.710091 & 0.0354475 & -0.370779 \\
$\hat{\theta}_{2}$ & 0.0562692 & 0.0559381 & - \\
$\hat{\theta}_{3}$ & - & 0.000649 & - \\
$\widehat{\mu}$ & 0.000798 & & 0.000724 \\
\hline
\end{tabular}


Model Checking: Before the interpretation and use of the model, we are to look at some tests to check whether the model is specified correctly. The following tests are applied to the residuals:

(i) Test for autocorrelation and partial autocorrelation.

(ii) Portmanteau test for residual autocorrelation.

(iii) Jarque Bera test for non normality.

Autocorrelation and Partial Autocorrelation of Residuals: In testing the autocorrelation and partial autocorrelation, if the residual is uncorrelated, then the result is adequate. Figures 4-6 shows that there is no serial correlation observed in the residuals of the variables, since all the series are within the $95 \%$ confidence intervals except in ARFIMA $(1,0.6238841,2)$.
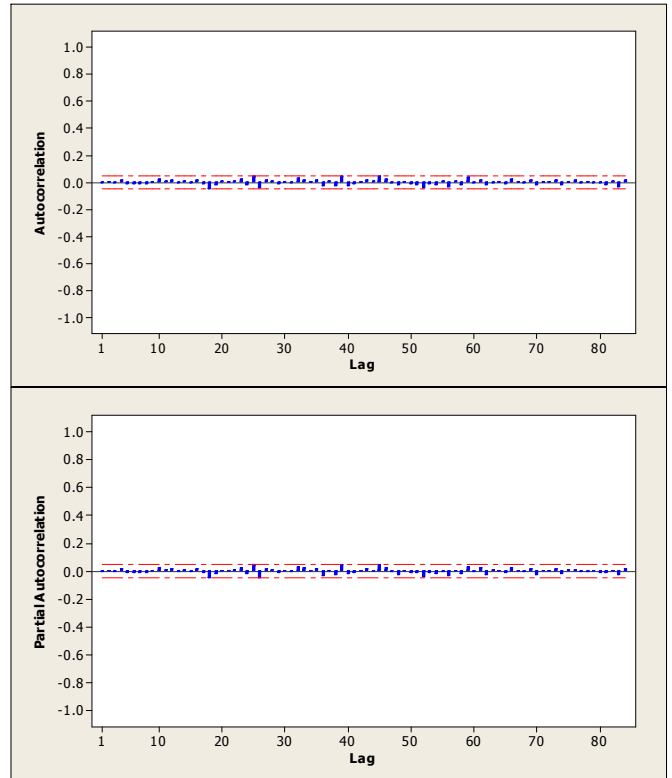

Figure 4: ARFIMA (3, 0.6238841, 1) Residual $\mathrm{ACF}$ and $\mathrm{PACF}$
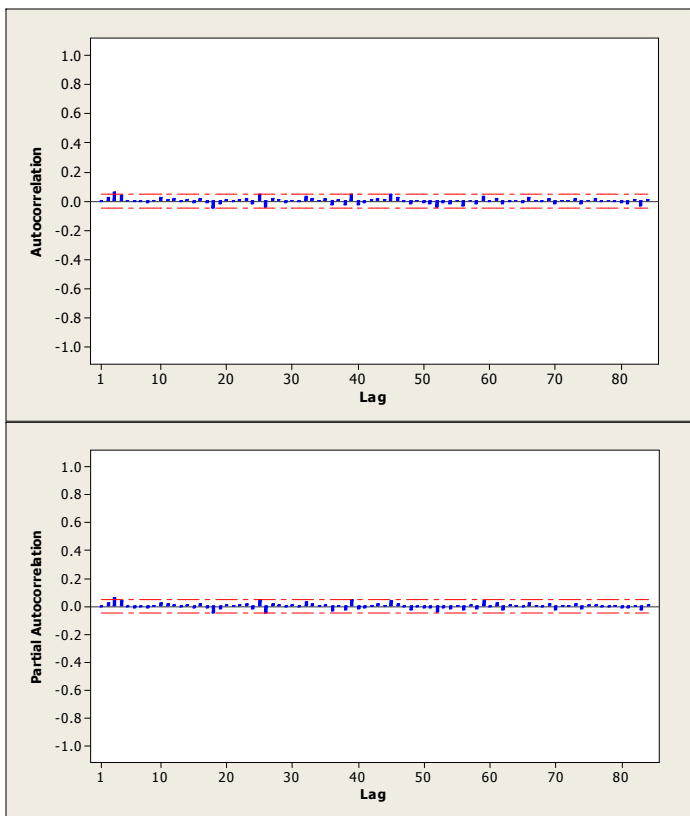

Figure 5: ARFIMA $(1,0.6238841,2)$ Residual $\mathrm{ACF}$ and $\mathrm{PACF}$
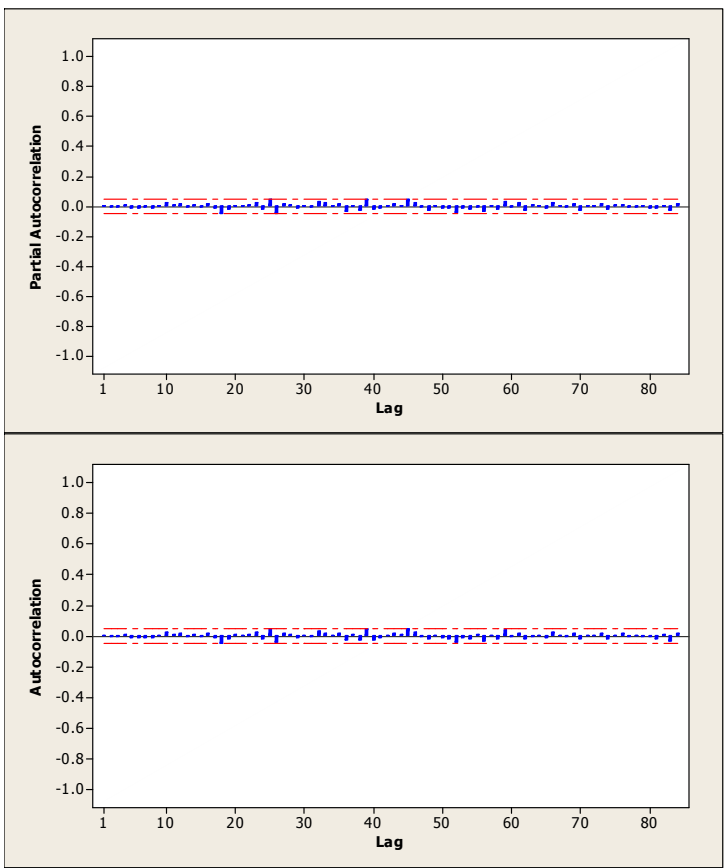

Figure 6: ARFIMA(1, 0.6238841, 3) Residual ACF and PACF 
We formally confirm the presence and absence of autocorrelation in the variables by portmanteau test. The results for the test are presented in Tables 7-8.

The null hypothesis of no autocorrelation can be accepted in Tables $7-8$. We conclude that there is no correlation in ARFIMA $(1,0.6238841,3)$ and ARFIMA $(3,0.6238841,1)$ models residual.

Test for Normality of Residual: The normality test of the residuals was performed to check if the residuals are normally distributed. The most popular method of checking normality is the Jarque-Bera test. The test statistic measures the difference of the skewness and kurtosis of the series with those from the normal distribution.

Under the null, the Jarque-Bera statistic is distributed as $\chi^{2}(2)$ (Luthkepohl and Kratzig, 2004).

Tables 9 - 10 indicate that the residuals of the models are normally distributed because all the $p$ values are greater than the critical value of 0.05

Table 7: Results of ARFIMA $(1,0.6238841,2)$ portmanteau test for autocorrelation of residual

\begin{tabular}{llll}
\hline Lags & Chi-square & DF & P-value \\
\hline 12 & 11.9 & 8 & 0.035 \\
24 & 18.8 & 20 & 0.537 \\
36 & 31.0 & 32 & 0.519 \\
48 & 42.3 & 44 & 0.544 \\
\hline
\end{tabular}

Table 8: Results of ARFIMA $(1,0.6238841,3)$ portmanteau test for autocorrelation of residual

\begin{tabular}{llll}
\hline Lags & Chi-square & DF & p-value \\
\hline 12 & 2.6 & 7 & 0.916 \\
24 & 10.2 & 19 & 0.948 \\
36 & 22.2 & 31 & 0.875 \\
48 & 34.0 & 43 & 0.837 \\
\hline
\end{tabular}

Table 9: Result for ARFIMA (3, $0.6238841,1)$ model Jarque-Bera test for normality of residual

\begin{tabular}{llll}
\hline Variables & Test statistic & DF & P-value \\
\hline DAT & 0.3747 & 2 & 0.8292 \\
\hline
\end{tabular}

Table 10: Result for ARFIMA(1,0.6238841,3) model Jarque-Bera test for normality of residual

\begin{tabular}{llll}
\hline Variables & Test statistic & DF & P-value \\
\hline DAT & 0.3746 & 2 & 0.8292 \\
\hline
\end{tabular}

Forecast: After good ARFIMA (p, d, q) model have been gotten, the next is to see its ability to forecast. The ability to do so will further testify the validity of the model. What we intend to do is to compare the forecast and the actual values and choose among, the one with the minimum error as our optimal model. The smaller the values of the error, the better the forecasting performance of the model (Olanrewaju and Olaoluwa, 2009). Tables $11-12$ are the ten days interval forecast for the models.

Table 11: Forecast for ARFIMA $(1,0.6238841,3)$

\begin{tabular}{llllll}
\hline Period & Forecast & Lower & Upper & Std.error & Actual \\
\hline 1544 & 0.04266 & -4.80029 & 4.88561 & 2.4670 & -2.89703 \\
1545 & -0.01411 & -4.85706 & 4.82884 & 2.4726 & -3.44864 \\
1546 & 0.01403 & -4.82892 & 4.85698 & 2.5193 & -1.95303 \\
1547 & -0.01026 & -4.85332 & 4.83281 & 2.5270 & -1.24712 \\
1548 & 0.00980 & -4.83335 & 4.83231 & 2.5283 & 2.84972 \\
1549 & -0.00676 & -4.84996 & 4.83644 & 2.5285 & 1.79135 \\
1550 & 0.00691 & -4.83633 & 4.85015 & 2.5285 & -3.36299 \\
1551 & -0.00438 & -4.84764 & 4.83889 & 2.5285 & -1.10714 \\
1552 & 0.00494 & -4.84605 & 4.84823 & 2.5285 & -3.50397 \\
1553 & -0.00275 & -4.84605 & 4.84054 & 2.5285 & 1.68969 \\
\hline
\end{tabular}


Table 12: Forecast for ARFIMA (3, $0.6238841,1)$

\begin{tabular}{llllll}
\hline Period & Forecast & Lower & Upper & Std.error & Actual \\
\hline 1544 & -0.20117 & -5.04519 & 4.64285 & 2.4673 & -3.10240 \\
1545 & -0.21925 & -5.06340 & 4.62491 & 2.4685 & -3.63560 \\
1546 & -0.08539 & -4.93186 & 4.76108 & 2.4738 & -2.08930 \\
1547 & -0.02818 & -4.88512 & 4.82875 & 2.4746 & -1.54740 \\
1548 & -0.00386 & -4.86225 & 4.85452 & 2.4748 & 2.49910 \\
1549 & 0.01424 & -4.84455 & 4.87302 & 2.4749 & 1.49430 \\
1550 & 0.02477 & -4.83422 & 4.88376 & 2.4749 & -3.44430 \\
1551 & 0.03054 & -4.82851 & 4.89305 & 2.4750 & -0.86500 \\
1552 & 0.03399 & -4.82508 & 4.89305 & 2.4750 & -3.39220 \\
1553 & 0.03601 & -4.82306 & 4.89509 & 2.4750 & 1.56020 \\
\hline
\end{tabular}

From the results in Tables 11-12, ARFIMA (3, $0.6238841,1)$ is selected as the optimal model because by comparing the forecast and the actual values, it was observed that the model ARFIMA $(3,0.6238841,1)$ has minimum error.

\section{CONCLUSION}

In this research, the stochastic structure of the daily average temperature data of Sokoto (DAT) has been analyzed by using fractionally integrated processes. In doing so, a much richer dynamic behavior of the series has been captured, not achieved by the classical $\mathrm{I}(1) / \mathrm{I}(0)$ representations but by fractional integration. We implement the method proposed by Geweke and Porter-Hudak (1983), to test and estimate $d$ under few prior assumptions concerning the spectral density of a time series. A number of heuristic methods to estimate self similarity parameter $\mathrm{H}$ have been presented and applied to the data and observed the estimated $\mathrm{H}$. We are able to show that the temperature series of Sokoto metropolis is fractionally integrated and has persistence dependence, therefore exhibits long memory.

Finally, the fractionally differenced DAT is taken and modeled using the Box-Jenkins approach. The correct model for the daily average temperature data (DAT) of Sokoto metropolis was built. Two models were found to be more adequate for describing, explaining and forecasting the temperature ARFIMA (3, 0.6238841, 1) and ARFIMA (1, 0.6238841, 3). But by checking the forecastability, ARFIMA $(3,0.6238841,1)$ model is the best optimal model that will best forecast Sokoto metropolis temperature.
The fitted model should be used for future forecast of temperature of Sokoto metropolis. Forecasting temperature is important to Agriculturist, Geographers and Hydrologist. Air temperature determines the rate of evaportranspiration.

\section{REFERENCES}

Akaike, H. (1974). Maximum Likelihood Identication of Gaussian Autoregressive Moving Average Models".Biometrika, 60(2): 255-265.

Baillie, R.T. (1996). Long memory processes and fractional integration in econometrics. $J$. Econometrics 73: 5 ï 9.

Baillie, R.T. and Kapetanios, G. (2007). Semi parametric estimation of long memory: The Holy grail or poisoned chalice. International Symposium on Financial Econometrics.

Beran, J. (1994). Statistics for Long-memory processes. London: Chapman \& Hall.

Box, G.E.P. and Jenkins, G.M. (1976). Time series analysis: forecasting and control, $2^{\text {nd }}$ Edn. San fransisco, CA: Holden-Day

Diebolt, C. and Guiraud, V. (2000). Long memory time series and fractional integration. A cliometric contribution to French and German economic and social history. Historical Social Res. 25: 4 -10.

Geweke, J. and Porter-Hudak, S. (1983). The estimation and application of long memory time series models. Time Series Anal, 4: 237

Gil-Alana, L.A. (2009). Persistence and time trends in the temperatures in Spain. Advances in Meteorology, 1-2. 
Gil-Alana, L.A. (2004). Long memory behaviour in the daily maximum and minimum temperatures in Melbourne, Australia. Royal Meteorological Soc. 11(4): 319-328.

Gil-Alana, L.A. (2005). Statistical modelling of the temperatures in the northern hemisphere using fractionally integrated techniques. J. Climate 18: 5357-5369.

Granger, C.W.J. (1980). Long memory relationships and the aggregation of dynamic models. J. Econometrics 14: 227238.

Granger. C. and Joyeaux (1980), "An Introduction to long memory time series models and Fractional differencing". J. Time Series Analy. 1: 15-19

Henry, M. and Zaffaroni, P. (2002). The long range dependence paradigm for macroeconomics and finance. http://www.econ.columbia.edu/RePEc/pdf/ DP0102-19.pdf.

Hosking, J.R. (1981). Fractional differencing. Biometrika 68: 165-178.

Hurst, H.E. (1951). Long term storage capacity for reservoirs. Transactions of the Am. Soc. Civil Engineers 116: 770-799.

Hurvich, C.M. and Deo, R.S. (1998). Plug-in selection of the number of frequencies in regression estimates of the memory parameter of a long memory time series. $J$. Time Series Analy. 3: 1-3.

Kwiatkowski, D., Phillips, P., Schmidt, P. and Shin, Y (1992). Testing the null hypothesis of stationarity against the alternative of a unit root: How sure are we that economic time series have a unit root? $J$. Econometrics 54: 159-178.
Laurini, M.P. and Portugal, S.P. (2003). Long memory in the R\$/US\$ exchange rate: $A$ robust analysisò Finance lab working paper,3.

Lutkepohl, H. (2005). New Introduction to Multiple Time Series Analysis, Springer, Berlin pp 51 - 55

Mandelbrot, B. and Van Ness, J.W. (1968). Fractional Brownian motion, fractional Brownian noise and application. SLAM Review, 10: 422-437.

Mayoral, L. (2007). A new minimum distance estimator for ARFIMA processes, Working Paper, Univeristat Pompeu Fabra

Odaki, M. (1993). On the Invertibility of fractionally differenced ARIMA processes. Biometrika 80: 703-709.

Olanrewaju, I.S. and Olaoluwa, S.Y. (2009). Measuring forecast performance of ARMA and ARFIMA models: An application to US Dollar/UK pound foreign exchange rate. Eur. J. Sci. Res. 32(2): 167-176.

Robinson, P.M. (1994). Semiparametric analysis of long-memory time series. Ann. Statist. 22: $515-539$.

Shimotsu, K. (2002). Exact local Whittle estimation of fractional integration with unknown mean and time trend. Working paper, Department of Economics University of Essex.

Sowell, F. (1992). Maximum likelihood estimation of stationary univariate fractionally integrated time series model. $J$. Econometrics 53: 165-188.

Yurekli, K. and Kurunc, A. (2007). Performances of Stochastic Approaches in Generating Low Stream flow Data for Drought Analysis. J. Special Hydrol. 5(1): 22-24. 